\title{
Impacts of COVID-19: Mitigation Efforts versus Herd Immunity
}

\section{CENTER FOR QUANTITATIVE ECONOMIC RESEARCH}

\section{CENTER FOR HUMAN CAPITAL STUDIES}

CENTER FOR FINANCIAL INNOVATION AND STABILITY

CENTER FOR HOUSING AND POLICY

\section{Karen Kopecky}

Federal Reserve Bank of Atlanta

\section{Tao Zha}

Federal Reserve Bank of Atlanta

\section{Summary:}

The rapid spread of COVID-19 is having devastating effects on the global economy. Governments around the world have been forced to pursue lockdown policies in an attempt to stem the spread of this deadly disease and bend the death curve. With death curves beginning to bend, governments will soon need to determine when and how to relax lockdown measures. The crucial question is: what are the consequences of reopening the economy? In this article, we address this question by studying both the model and the data and discuss the challenges we face moving forward.

\section{Key findings:}

1. The observed decline in daily deaths could be due to two scenarios: lockdown policies and herd immunity.

2. Both the model used by epidemiology experts and the data collected thus far cannot distinguish these two scenarios.

3. Comprehensive testing can help resolve this uncertainty by quickly and accurately identifying new cases so that future outbreaks could be contained by isolation and contact tracing measures.

JEL classification: I1, E6, H12

Key words: COVID-19, identification problem, death curve, lockdown, social distancing, herd immunity, reopening the economy, testing

https://doi.org/10.29338/ph2020-03

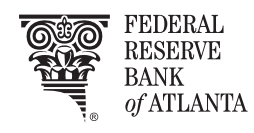

The Federal Reserve Bank of Atlanta's Policy Hub

leverages the expertise of Atlanta Fed economists and researchers to address issues of broad policy interest. Our research centers coordinate this work and seek to influence policy discussions. Areas of interest include: forecasting, fiscal policy, and macroeconomics (Center for Quantitative Economic Research); financial stability, innovation, and regulation (Center for Financial Innovation and Stability); human capital, labor markets, health, and education (Center for Human Capital Studies); and government-sponsored entity reform, mortgage markets, and affordable housing (Center for Housing and Policy). Sign up for email updates at frbatlanta. org/research/publications/policy-hub. 


\section{Impacts of COVID-19: Mitigation Efforts versus Herd Immunity}

Abstract: The rapid spread of COVID-19 is having devastating effects on the global economy. With death curves beginning to bend, governments will soon need to determine when and how to relax lockdown measures. The crucial question is: what are the public health consequences of reopening the economy? In this article, we argue that the observed decline in daily deaths could be due to two scenarios: social distancing measures and herd immunity. Both the widely used SIR model and the data collected thus far cannot distinguish these two scenarios. Such an identification problem generates a large degree of uncertainty about the public health consequences of restarting the economy. Comprehensive testing can help resolve this uncertainty by quickly and accurately identifying new cases so that future outbreaks could be contained by isolation and contact tracing measures.

About the authors: Karen Kopecky is a research economist and associate adviser on the macroeconomics and monetary policy team in the Research Department at the Federal Reserve Bank of Atlanta and an adjunct professor of economics at Emory University. Tao Zha is executive director of the Center for Quantitative Economic Research in the research department of the Federal Reserve Bank of Atlanta and a professor of economics at Emory University.

JEL classification: I1, E6, H12

Key words: Covid-19, identification problem, death curve, lockdown, social distancing, herd immunity, reopening the economy, testing

Acknowledgments: The authors are grateful to Paula Tkac for her detailed comments and suggestions. They would also like to thank Andy Atkeson, Salomé Baslandze, Chris Cunningham, Nikolay Gospodinov, Eric Leeper, and Ed Nosal for helpful comments and discussions. The views expressed here are the authors' and not necessarily those of the Federal Reserve Bank of Atlanta or the Federal Reserve System. Any remaining errors are the authors' responsibility.

Comments to the authors are welcome at karenakopecky@gmail.com and zmail@tzha.net. 
According to a recent update of the Institute for Health Metrics and Evaluation's (IHME) model, the United States is past the peak in estimated daily deaths due to COVID-19. While the model predicts that roughly 40,000 more U.S. residents will die before August 4, 2020, it appears that the death rate is slowing and the "death curve" has begun to bend (represented by the dashed lines in figure 1). Data for the UK, Spain, Italy, and Germany seem to be following a similar pattern. If the model's predictions are accurate, this is decidedly good news. Local and national governments around the world are now turning their attention to when and how to relax social distancing measures. The IHME model assumes that social distancing measures will remain in place for the next four months and thus can be seen as a benchmark against which to measure a change in policy. A crucial question facing policymakers is: what are the public health consequences of relaxing social distancing measures and "reopening the economy"? Will the death rate begin to rise if social distancing measures are rescinded?

FiguRE 1.

Total deaths

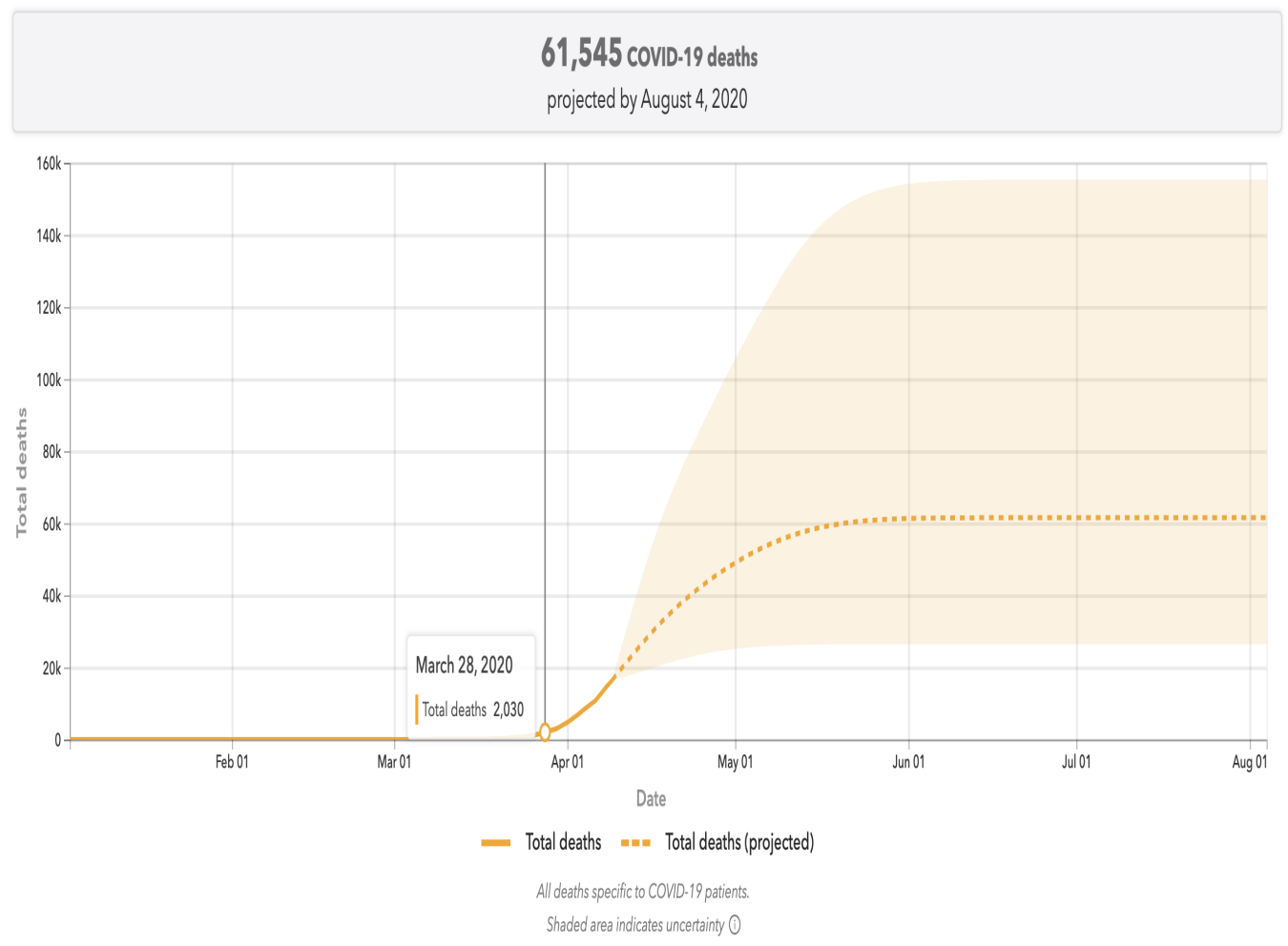

Note: Data indicate the April 9, 2020, forecast from the IHME.

The answer to this question depends on how effective social distancing policies have been at reducing the transmission rate of the virus. If they have been very effective, 
the observed decline in daily deaths is likely due to the social distancing policies directly. However, it is also possible that in the United States and elsewhere the reason for the decline in daily deaths is that we are close to achieving herd immunity (Barry, 2009; Atkeson, 2020; Correia, Luck, and Verner, 2020; Stock, Aspelund, Droste, and Walker, 2020). By herd immunity we mean that COVID-19 is widespread enough that the rate of additional spread is starting to decline.

If the cause for the decline is mainly social distancing policies, and if we have not achieved herd immunity, then reopening businesses may lead to additional severe outbreaks of COVID-19. This sort of subsequent outbreak was the experience in the United Kingdom during the 1918 Spanish Flu pandemic (figure 2). The extent to which the loosening of official lockdown policies increases transmission is of course determined by the private actions of citizens. Citizens who have been practicing social distancing and sheltering at home may be reluctant to resume their normal social and work activities if they still fear infection. This behavior would tend to limit transmission of the virus relative to normal, prepandemic conditions. Alternatively, if the decline in daily deaths is mainly due to herd immunity, a return to normal economic activity would be less likely to result in widespread outbreaks.

FiguRE 2.

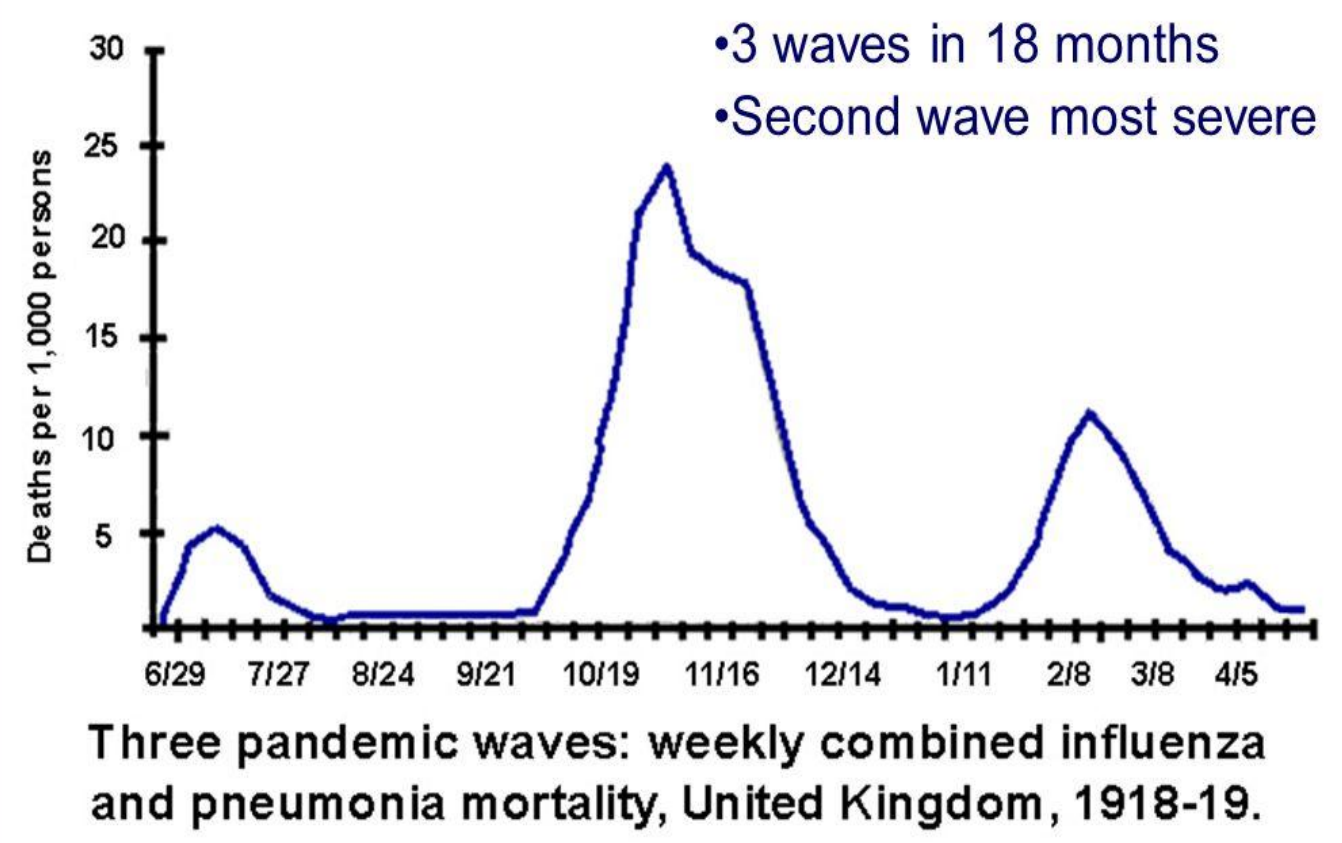

Source: Taubenberger, EID 2006. Jordan, AMA 1927

Note: Data depict the risk of a second (and even third) wave if lockdown is lifted too soon. 
Uncertainty about which of these scenarios we are in has generated enormous disagreement among epidemiologists (Ioannidis, 2020 Bendavid and Bhattacharya, 2020; Silverman, Hupert, and Washburne, 2020). Figure 3 shows the telling results from a survey of epidemiology experts conducted by the University of Massachusetts Amherst (McAndrew, 2020). Unfortunately, neither the currently available data nor the mathematical model used by epidemiologists will allow policymakers to distinguish between these two alternative scenarios.

\section{FIGURE 3.}

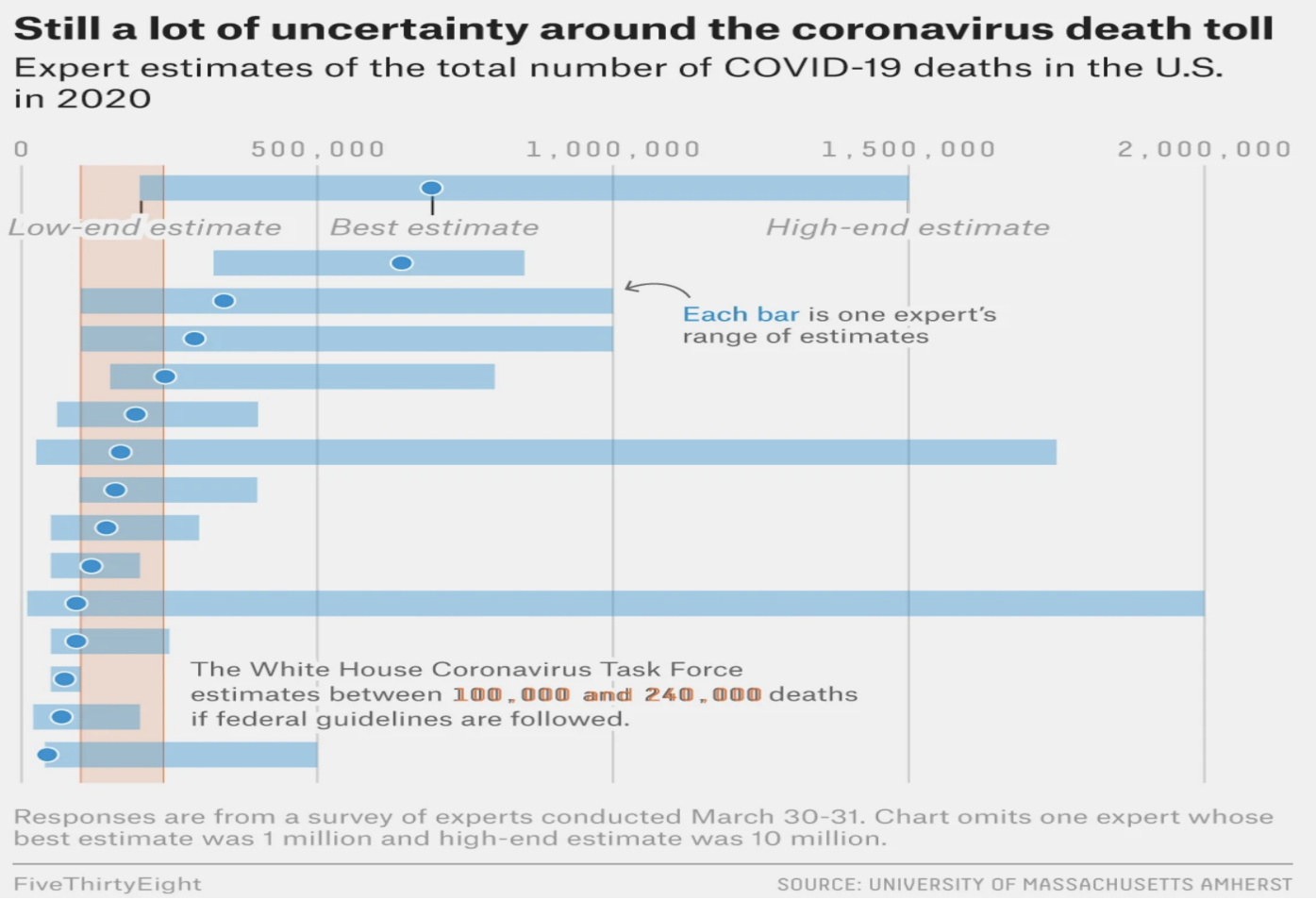

Note: Survey conducted on March 30-31, 2020, on the total death toll.

\section{THE SIR MODEL}

The mathematical model widely used by epidemiologists to model the spread of COVID-19 is called the Susceptible Infectious Recovered (SIR) model. In its simplest form, this model divides the population of people into three different subgroups. The susceptible group $(S)$ consists of people who have not yet been exposed to the virus, have no immunity to it, and are thus susceptive to infection. The infected group $(I)$ are people who are actively infected and contagious. If a person from group $I$ meets a person from group $S$, there is some risk the infected person will transmit the virus to the susceptible person. The third group is the recovered group $(R)$. These 
are people who previously had the virus and are now assumed to be immune and no longer contagious. The standard SIR model categorizes those who do not survive infections with the virus into group $R$ since they are also no longer able to infect others. We modify this standard model to include only individuals who survive and recover from the virus in group $R$ and create a new group $(D)$ that consists of those for whom the virus is fatal.

Figure 4 presents a flow chart of the model that shows how individuals can move across different groups as they become infected with the virus. The variables $S, I, R$, and $D$ represent the number of people in each group. The infection rate is the rate at which people in the susceptible group interact with a member of the infected group and become infected themselves. The recovery and fatality rates are the rates at which infected people either recover or die from the virus.

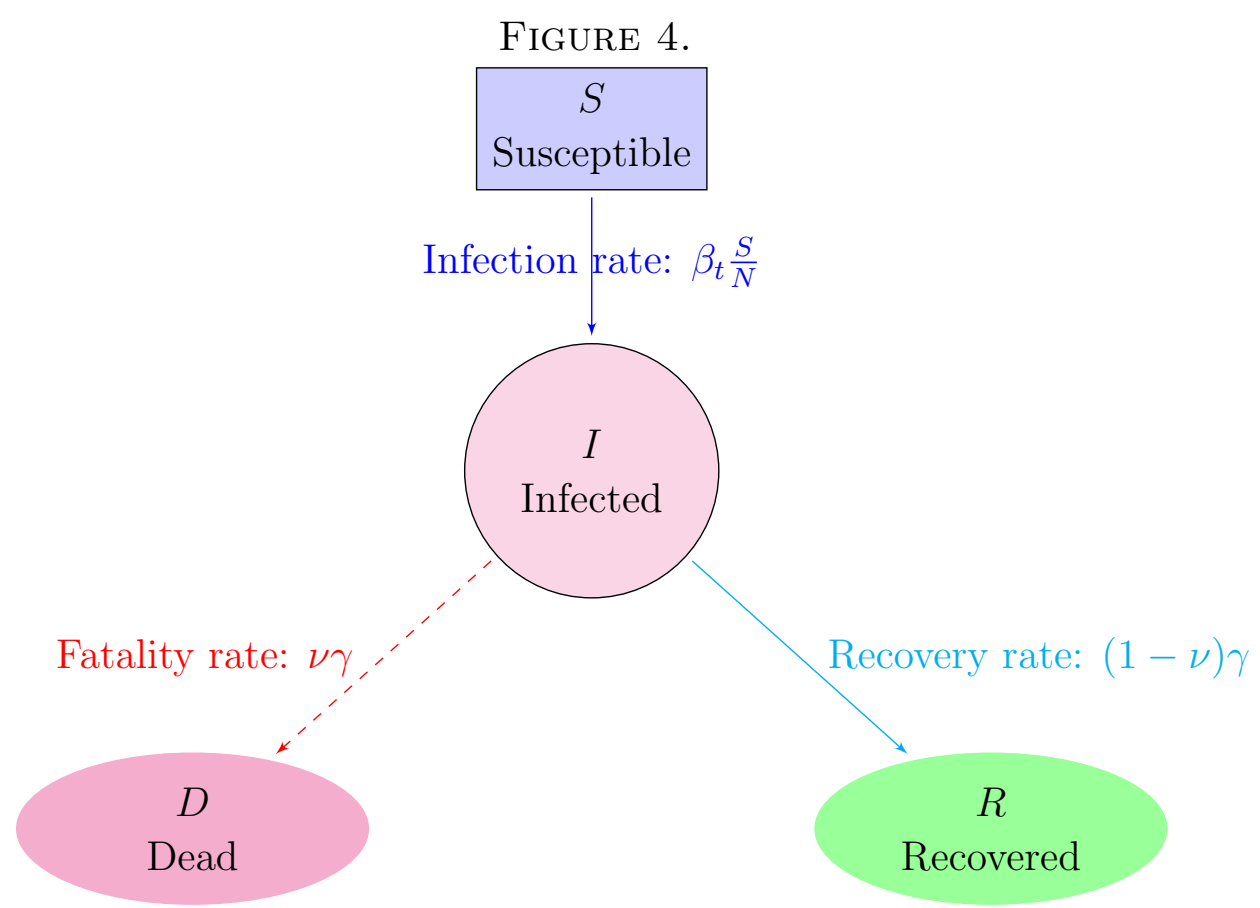

An important assumption of the model is that people in the population are randomly meeting one another. If the total number of people in the population is $N$, the probability an infected person meets a susceptible person is just the fraction of susceptible people in the population $\frac{S}{N}$. We will use the symbol $\beta_{t}$ to denote the transmission rate of the virus, or the probability that a meeting between an infected and susceptible person results in a new infection. The transmission rate also captures the frequency of meetings between infected and susceptible people. Putting these two ideas together, we see that the rate of new infections is $\beta_{t} \frac{S}{N}$. Simultaneously, already 
infected individuals are transitioning to recovered or fatality status at rate $\nu$. Therefore, combining the inflow of newly infected individuals with the outflow of recoveries and fatalities, we can study how the number of infected individuals changes over time (see the following mathematical equation).

$$
d I / d t=\beta_{t} \frac{S}{N} I-\gamma I=\left(\frac{\beta_{t}}{\gamma} \frac{S}{N}-1\right) \gamma I .
$$

Consider first the exit rate, which is the rate at which infected people either recover or die. This rate is calibrated to be the inverse of the contagious (infected) period, which is approximately 18 to 25 days. Assume that the fraction of infected people who die is $\nu$. Then, the fatality rate conditional on infection is $\nu \gamma$ and the recovery rate (also conditional on infection) is $(1-\nu) \gamma$.

Whether or not the number of infected people is increasing or decreasing at any time $t$ will depend on the net effect of these two forces, which can be summarized by the reproductive ratio $\mathcal{R}_{t}=\frac{\beta_{t}}{\gamma} \frac{S}{N}$. Notice, from the far right-hand side of the above equation, that if $\mathcal{R}_{t}>1$, the number of infected people, $I$, will increase over time, while if $\mathcal{R}_{t}<1$, it will decrease. Early in the epidemic, nearly everyone is susceptible to the virus, and $\frac{S}{N}$ is close to 1 . Over time, however, as more and more initially susceptible people become infected, $S / N$ will decline. Notice that as $S / N$ declines so does $\mathcal{R}_{t}$. Herd immunity occurs when $S / N$ becomes so small that $\mathcal{R}_{t}$ falls below 1 . At this point, so many people have already been exposed to the disease (and are thus immune), that meetings between infected and susceptible people become too rare for the number of infected people in the population to continue to increase. In other words, once herd immunity is achieved, the number of infected people in the population begins to decline. Assuming that a constant fraction of infections will result in fatality, as the number of infections declines, the number of daily deaths also starts to decline. This decline in the number of daily deaths is referred to as the "bending" of the cumulative death curve. This does not mean, however, that when we see a decline in daily deaths we can assume that we are achieving herd immunity. A decline in the number of daily deaths can also be the result of effective mitigation policies and behaviors. Examples of such policies are mandatory social distancing, shelter-in-place edicts that minimize social contact, and recommendations that masks be worn in public areas. Individuals may also engage in voluntary mitigation behaviors such as hand washing, wearing gloves, grocery delivery, etc. These types of policies reduce the transmission rate of the virus, $\beta_{t}$. To illustrate the difficulty in differentiating between herd immunity and a fall in the transmission rate, consider two scenarios for the SIR model illustrated in figure 5. The scenario shown in the left chart assumes a permanent decline in the virus transmission rate at day 21 due to effective mitigation policies. The mitigation policies lower the 
transmission rate from infected to susceptible individuals and the number of infections begins to decline.

\section{FIGURE 5.}
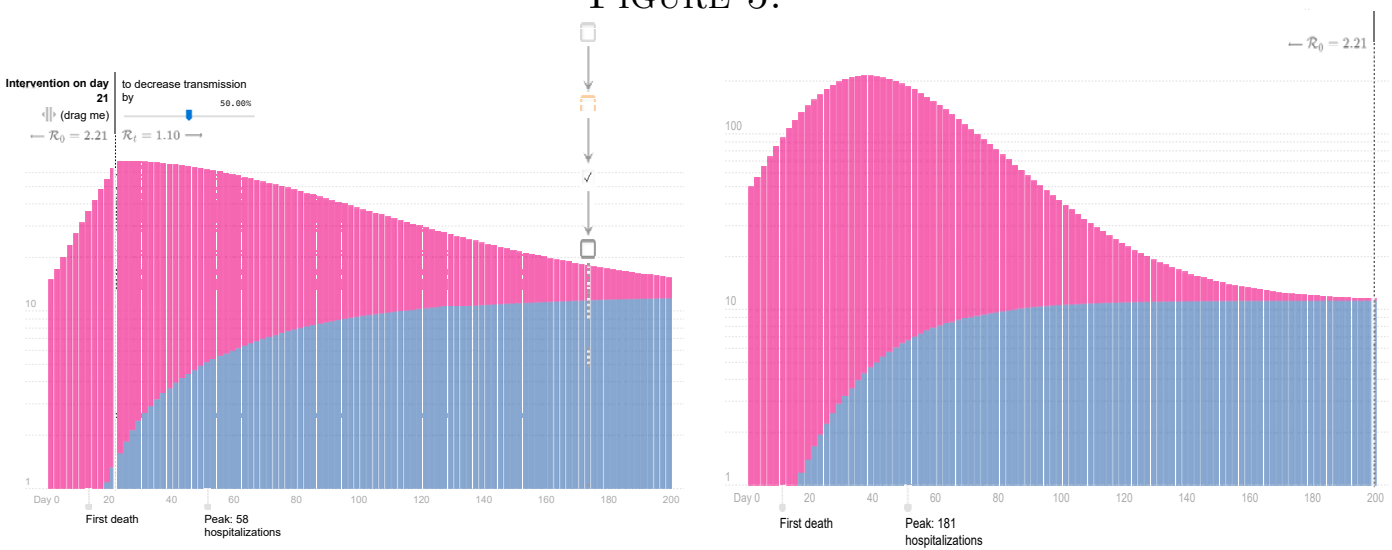

Note: These figures were generated using the Epidemic Calculator available at https://gabgoh.github.io/COVID/index.html

The scenario shown in the right chart assumes that no mitigation policies are put into effect, so no change occurs in the transmission rate of the virus over time. In this scenario the only factor pushing $\mathcal{R}_{t}$ down over time is the declining value of $\frac{S}{N}$. Under both scenarios, the SIR model produces a similar pattern of cumulative deaths over time as shown by the nearly identical blue curves. The figure shows that it is hard to distinguish between herd immunity and effective mitigation policies just by looking at the pattern of deaths over time. Essentially, a lower transmission rate or a lower fraction of the population that is susceptible can produce observationally equivalent death patterns. Notice, however, that the two scenarios have different implications for the number of infected individuals in the population (the pink curves in the figures). If declines in the cumulative death rate are driven by herd immunity, it suggests that more people in the population have already been infected with the virus.

If we had accurate data on the total number of infections and deaths, we would know the true fatality rate of the virus and be able to determine whether herd immunity or mitigation policies were driving the declining number of deaths. Unfortunately such data do not exist. Without widespread testing, measuring the number of people infected with COVID-19 is extremely difficult as there is increasing evidence that COVID-19 has a high asymptomatic rate. The asymptomatic rate is the fraction of contagious people who have either no symptoms or symptoms mild enough to be confused with a common cold and not reported (Stock, 2020). But how high is the asymptomatic rate exactly? The answer to this question is important because the higher the asymptomatic rate, the lower is the value of $\frac{S}{N}$ and the closer we are to achieving herd immunity. 
In many countries, mortality rates have been declining over time (see figure 6), but we do not know whether these declines are due to effective mitigation policies or herd immunity. When the death curve begins to bend, the SIR model is unable to distinguish between these two factors. This problem is what economists often call an identification issue.

\section{FiguRE 6.}

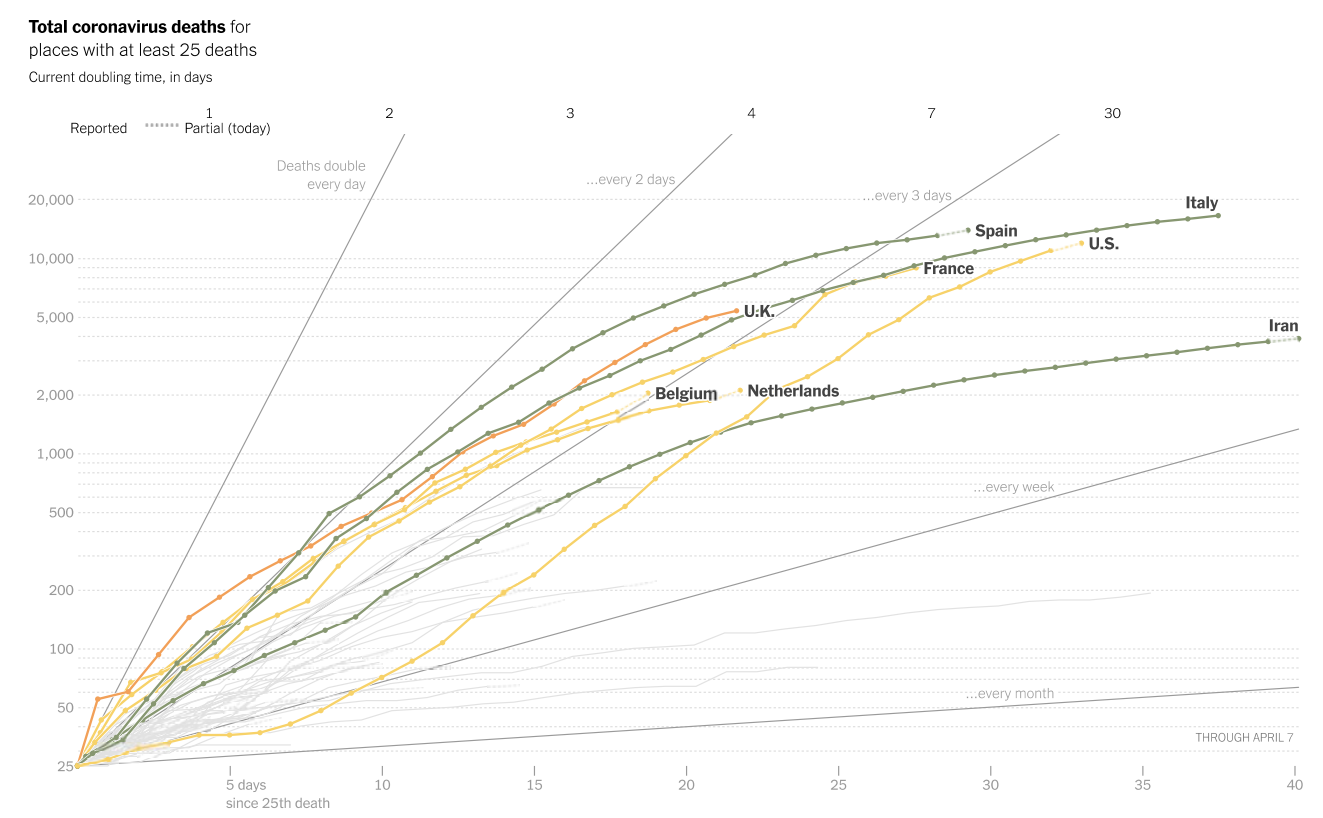

Note: Data depicted death curves across countries. Source: Katz and Sanger-Katz (2020) "Coronavirus Deaths by U.S. State and Country Over Time: Daily Tracker," New York Times.

\section{UPPER-TAIL UNCERTAINTY}

The identification problem described above has led to wide dispersion in epidemiologists' most likely forecasts. It has also led to substantial upper-tail uncertainty in individual forecasts. Figure 3 shows the long upper tail of the distribution of deaths displayed by each bar. The upper-tail uncertainty is so enormous that a few experts are predicting that the death toll may exceed a million.

This uncertainty surrounding the high-end estimates of deaths might reflect epidemiologists' inability to ascertain both the degree to which mitigation policies will slow the transmission rate and distinguish mitigation effects from the effects of herd immunity. Although mitigation policies have been in place, "there is significant uncertainty over how well people will comply with these directives and how much they 
actually reduce transmission, considering all of the activities that are exempted as essential.' ' In this very same survey, the range of estimates of unreported infections was so wide that the estimated number of unreported cases could easily end up in the multimillions (see figure 7). The epidemiology experts in this survey believed that only 12 percent of all infected cases have been reported, but there is enormous uncertainty over this estimate, as figure 7 demonstrates. As explained above, unreported cases make it very difficult to calibrate the SIR model.

\section{FiguRE 7.}

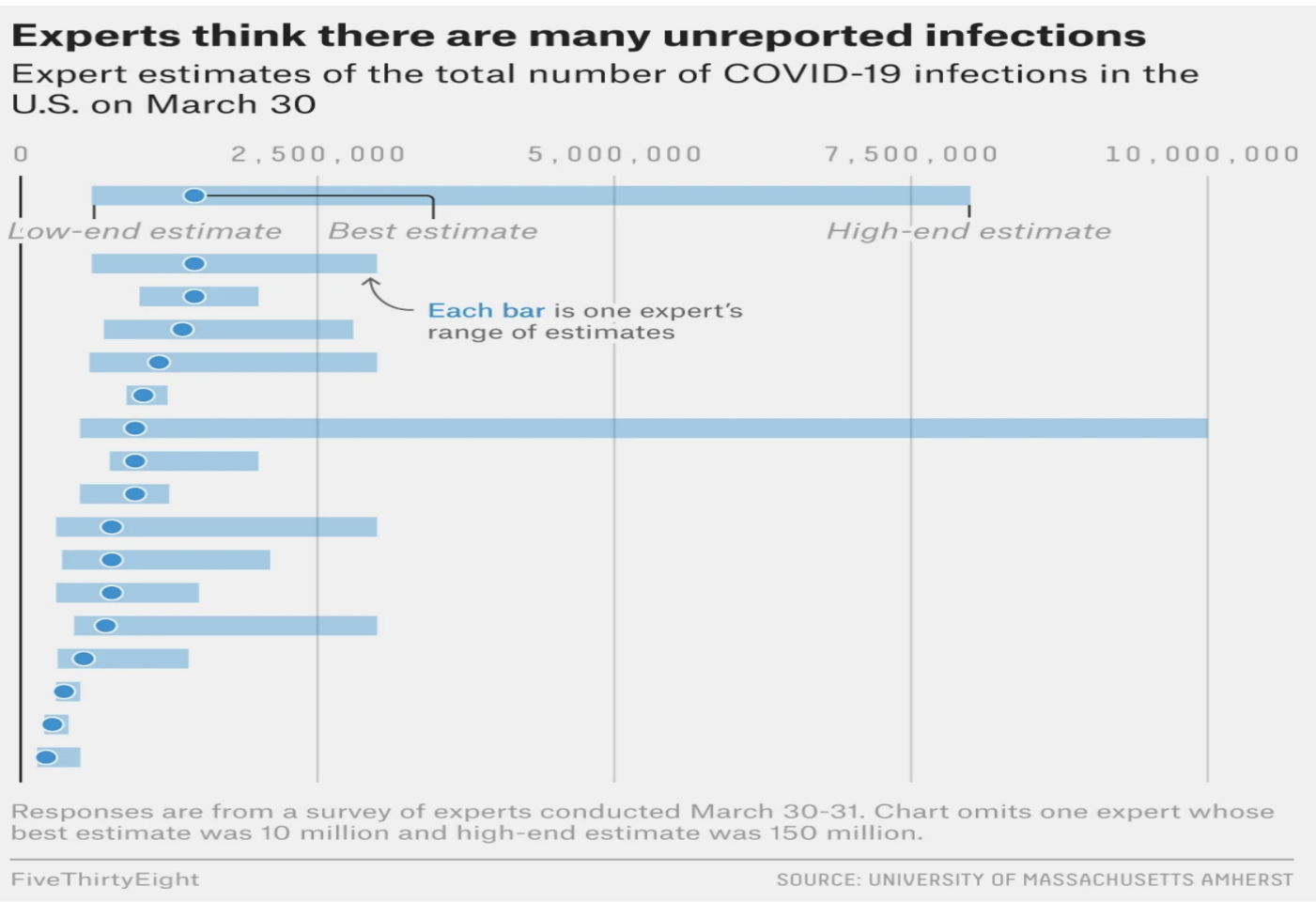

Note: Data represent results of a survey of epidemiology experts, conducted on March 3031, 2020, on the number of unreported infectious cases.

Similarly, the upper-tail uncertainty shows up in the death forecasts often used by the U.S. government. Although the most likely path of predicted total deaths was adjusted downward from the initial forecasts, the high-end estimate of total deaths changed little, which translates to uncertainty over which scenario we are in when death rates decline.

\footnotetext{
${ }^{1}$ See the article at <https://fivethirtyeight.com/features/best-case-and-worst-case-coronavirusforecasts-are-very-far-apart/ $>$ (accessed April 16, 2020).
} 


\section{CONCLUDING REMARKS}

Dr. Anthony Fauci, director of the National Institute of Allergy and Infectious Diseases and the White House health adviser, warned that when governments begin to relax restrictions, new cases are likely to occur and the "question is how do you respond to them?"'2 World Health Organization officials have recently indicated that "not all people who recover from the coronavirus have the antibodies to fight a second infection.' 3 This new discovery implies that there may still be a long way to go before we achieve herd immunity. In such a case, extensive testing can help reduce the reproductive ratio. If testing becomes more readily available, new cases can be quickly and accurately identified and any future outbreaks could be contained by isolation and contact tracing measures. With aggressive containment efforts, governments could restart the economy and still keep the reproductive ratio low enough to prevent overburdening the healthcare system. However, without frequent and extensive testing, reopening the economy en masse could have disastrous consequences. If we are still far from achieving herd immunity, we could experience a second wave of infections even larger than the first.

Despite the difficulties in disentangling the impact of mitigation policies currently in place from herd immunity and the uncertainty it implies, local and national governments are moving forward with plans to reopen their economies. For instance, Germany plans to reopen on Monday, April 20.4 In the United States, the Centers for Disease Control and the Federal Emergency Management Administration have released a guide to reopening parts of the country, and several U.S. state governors are coordinating to develop reopening strategies $\left.\right|^{5}$ Relaxing mitigation polices under so much uncertainty about their implications will be challenging but at the same time informative. The impact of removing lockdown, even gradually, on hospitalizations and deaths will be revealing about its relative importance in reducing the infection and death rates.

In the absence of widespread testing in the United States., there may also be other ways to distinguish between herd immunity and mitigation policies and reduce the uncertainty in forecasts. For instance, experts may be able to use data from other

\footnotetext{
${ }^{2}$ See the article at <https://www.cnbc.com/2020/04/10/trump-says-hes-not-going-to-reopeneconomy-until-we-know-this-country-is-going-to-be-healthy.html> (accessed April 16, 2020).

${ }^{3}$ See the article at <https://www.cnbc.com/2020/04/13/who-officials-say-its-unclear-whetherrecovered-coronavirus-patients-are-immune-to-second-infection.html > (accessed April 16, 2020).

${ }^{4}$ See the article at <https://www.wsj.com/articles/germany-to-begin-gradually-reopening-itseconomy-next-week-11586989014> (accessed April 16, 2020).

${ }^{5}$ See the article at <https://www.washingtonpost.com/health/2020/04/14/cdc-fema-havecreated-plan-reopen-america-heres-what-it-says/> and <http://www.oleantimesherald. $\mathrm{com} /$ news/state/cuomo-coalition-of-6-northeast-states-set-to-announceregional-reopening-plan/article_6fe305a6-2abc-566d-b561-04fc74899d4d. html> (accessed April 16, 2020).
} 
FEDERAL RESERVE BANK OF ATLANTA POLICY HUB $\diamond$ NO. 03-2020 $\diamond$ APRIL 202011

countries, such as Iceland, that have extensively tested their populations to improve their predictions about the evolution of the virus in the U.S.6.$^{6}$ With time, researchers may be able to use statistical techniques that allow these data to reduce the uncertainty in the values of the parameters of the SIR model even without widespread testing and data on infections.

\footnotetext{
${ }^{6}$ See the article at <https://www.france24.com/en/20200415-study-shows-iceland-got-it-rightwith-early-widespread-virus-testing $>$ (accessed April 16, 2020).
} 
FEDERAL RESERVE BANK OF ATLANTA POLICY HUB $\diamond$ NO. 03-2020 $\diamond$ APRIL 202012

\section{REFERENCES}

Atkeson, A. (2020): "An Introduction to SEIR Models for COVID-19 Disease Scenarios," UCLA, Unpublished Slides.

BArry, J. M. (2009): "Pandemics: Avoiding the Mistakes of 1918," Nature, 459, $324-325$.

Bendavid, E., And J. Bhattacharya (2020): "Is the Coronavirus as Deadly as They Say?," Wall Street Journal, <https://www.wsj.com/articles/ is-the-coronavirus-as-deadly-as-they-say-11585088464>, March 24.

Correia, S., S. LuCK, and E. Verner (2020): "Pandemics Depress the Economy, Public Health Interventions Do Not: Evidence from the 1918 Flu," Federal Reserve Board and MIT, Unpublished Manuscript.

IoAnnidis, J. P. (2020): "A Fiasco in the Making? As the Coronavirus Pandemic Takes HOld, We Are Making Decisions Without Reliable Data," Stanford University, <https://www.statnews.com/2020/03/17/a-fiasco-in-the-making-asthe-coronavirus-pandemic-takes-hold-we-are-making-decisions-without-reliable$\operatorname{data} />$, March 17 .

McAndrew, T. (2020): "Preliminary Report on Aggregated Expert Predictions on COVID-19," University of Massachusetts Amherst, <https: / / works. bepress.com/mcandrew/2/>, March 18.

Silverman, J. D., N. Hupert, and A. D. Washburne (2020): "Using ILI Surveillance to Estimate State-specific Case Detection Rates and Forecast SARSCoV-2 Spread in the United States," medRxiv, BMJ Yale, <https : / / doi .org/ $10.1101 / 2020.04 .01 .20050542>$, April 14.

Stock, J. H. (2020): "Random Testing is Urgently Needed," Harvard University, <http://www.igmchicago.org/wp-content/uploads/2020/03/ Random-Testing-is-Urgently-Needed_IGM.pdf>, March 23.

Stock, J. H., K. M. Aspelund, M. Droste, and C. D. Walker (2020): "Estimates of the Undetected Rate among the SARS-CoV-2 Infected using Testing Data from Iceland," Harvard University and MIT, Unpublished Manuscript. 